\title{
Sagittal balance of the spine
}

\author{
J. C. Le Huec ${ }^{1} \cdot$ W. Thompson ${ }^{1} \cdot$ Y. Mohsinaly ${ }^{1} \cdot$ C. Barrey $^{1} \cdot$ A. Faundez ${ }^{1}$
}

Received: 8 September 2018 / Revised: 13 July 2019 / Accepted: 16 July 2019 / Published online: 22 July 2019

(c) The Author(s) 2019

\begin{abstract}
The static sagittal balance of the normal spine is a physiological alignment of the spine in the most efficient manner by the muscular forces. During gait, this balance is constantly thwarted by single-foot support. This analysis involves the study of parameters which are now well defined. The pelvic incidence is constant, and the sacral slope and the pelvic tilt are positional. The cervical parameters are the upper $(\mathrm{O}-\mathrm{C} 2)$ and lower cervical curvatures $(\mathrm{C} 2-\mathrm{C} 7)$, the $\mathrm{C} 7$ slope, the spinocranial angle and the vertical cervical offset. At the thoracic and lumbar level, they are, respectively, kyphosis and lordosis. The OD-HA (odontoid hip axis) angle is the most efficient parameter to analyse the global balance. The average values of these parameters are reported with the new 3D measurements by Le Huec et al. The relationship between these different parameters was analysed, and Roussouly proposed his classification of the different spine shape. Ageing makes it possible to show compensation mechanisms at three levels: spinal, pelvic and lower limbs. Understanding these different data allows for better planning of the surgical management of the patients. Global evaluation of the entire spine and the measurement of the aforementioned parameters allow to determine the extent of the correction to be performed during surgery. Taking these parameters into account also enables us to understand the complications involved in this type of surgery: transitional syndromes or junctional syndromes. Integration of these parameters into the study of gait is an area still under investigation.
\end{abstract}

\section{Graphic abstract}

These slides can be retrieved under Electronic Supplementary Material .
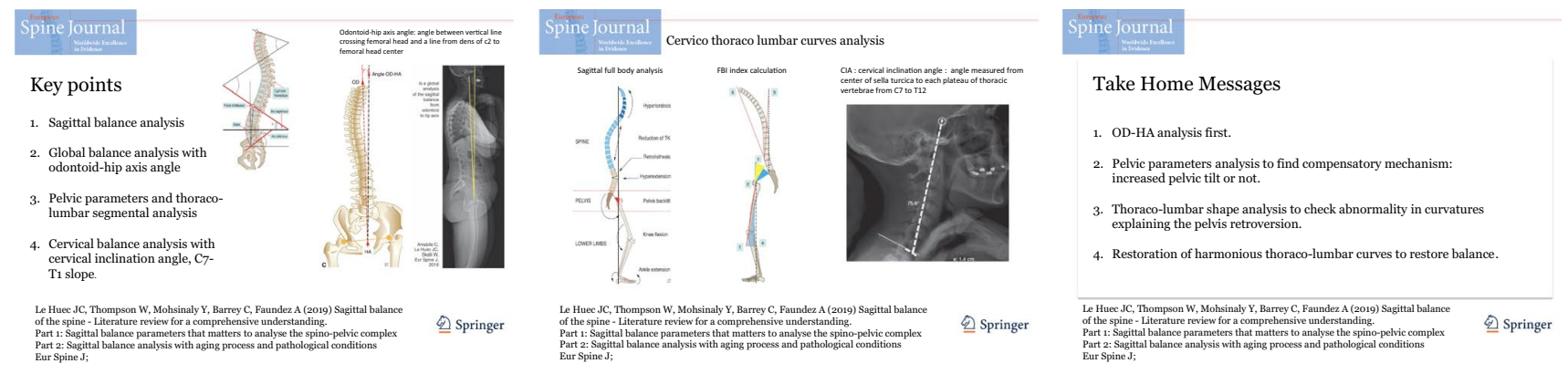

Keywords Sagittal balance $\cdot$ Spinal balance $\cdot$ Spinal imbalance $\cdot$ Junctional syndrome $\cdot$ Lumbar lordosis $\cdot$ Pelvic tilt . Global balance analysis $\cdot$ Spino-pelvic parameters $\cdot$ Spino-cranial angle $\cdot$ FBI

Electronic supplementary material The online version of this article (https://doi.org/10.1007/s00586-019-06083-1) contains supplementary material, which is available to authorized users.

J. C. Le Huec

jclehuec1@gmail.com

1 Spine Unit, Polyclinique Bordeaux Nord Aquitaine, Université bordeaux, 33000 Bordeaux, France

\section{Introduction}

Balance or equilibrium (from the Latin aequilibrium, from aequus "equal" and libra "balance, weight") is a concept that describes a situation in which the forces present are equal, or such that none surpasses the sum of the others. It is in this condition that the actions of the agonist and 
antagonistic muscles of the spine are minimized and therefore most efficient.

The human species is characterized by bipedalism, which has the advantage of freeing the upper limbs for other tasks, but this is done at the cost of a more precarious overall balance. Indeed, the great apes, unlike humans, move on all the four. Their spine presents a single thoraco-lumbar curvature in kyphosis. Their centre of mass is projected at the centre of their support polygon which is located in front of the footprint of the hind legs.

The hominids modified their profile by developing cervical curvature and lumbar lordosis to maintain a horizontal gaze and to free the upper limbs that are no longer carriers. To understand the conditions necessary for such a balance, it is important to analyse matters both statically and dynamically.

Indeed, during gait, bipedal static balance is constantly thwarted by single-foot support while stepping and must be compensated by a muscular tolerance which tends to reestablish this balance by passing through short phases of instability. As will be seen, sagittal balance is not limited to the analysis of static curves, as it requires consideration of more or less efficient compensation phenomena occurring during walking but also in static conditions.

The centre of mass should project as close as possible towards the centre of a reduced polygon situated between the two feet in a stable position and in an area of constant small rebalancing efforts. If the centre of mass tends to project outside this polygon, the rebalancing efforts become much more important, as described by Dubousset in his concept of "efficiency cone" (Fig. 1a, b) [1].

\section{Part I: Sagittal balance parameters that matter to analyse the spino-pelvic complex}

The purpose of this chapter is to describe the morphologic criteria that can be analysed to determine static sagittal alignment of the spine, and to then analyse the compensation phenomena which may be physiological or pathologic. The chapter also aims to use the parameters and algorithms that allow global analysis to provide therapeutic solutions adapted to each case.

\section{Fundamentals of sagittal alignment of the human body in a static vertical position}

A human being stands and moves in an environment subject to gravity. Its spine is subject to subsequent constraints. There is a close relationship between the pelvis and the spine due to this bipedal position. The upright posture is acquired by the enlargement and verticalization of the pelvis (Fig. 2a,

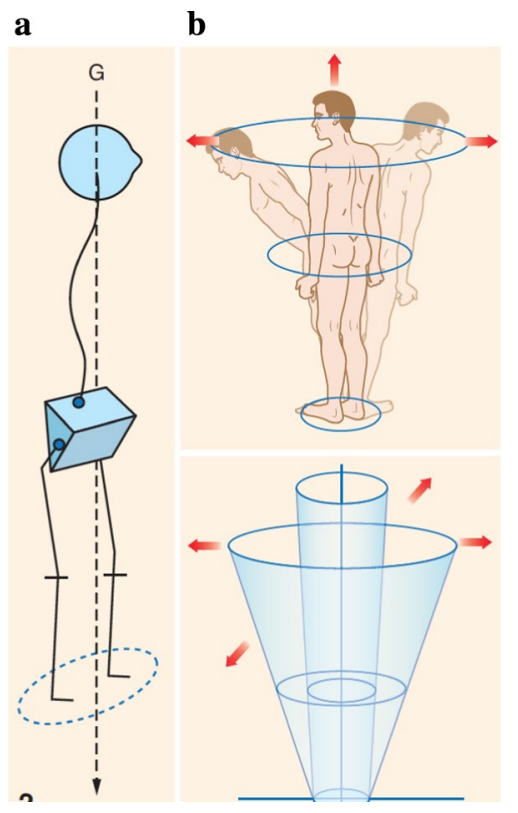

Fig. 1 a Efficiency conus of Dubousset. Illustration of the concept of the efficiency conus [1]. The larger the diameter of the base of the conus (located at the level of the head), the greater the effort that muscles should exert to keep the body upright. b The figure shows an internal smaller conus, which is the economic equilibrium conus, where the subject maintains a relaxed upright position. The external larger conus represents the position where the subject has to activate erector spinae muscles to maintain an upright position (relative equilibrium). The imbalance can then occur with a minimal increase in lateral bending effort when the patient is in this an area of relative equilibrium

b), leading to the characteristic curvatures of the vertebral column in sagittal plane. Unlike some species, such as chimpanzees, that adopt the erect position only occasionally, the latter is the preferred posture in humans. It must therefore be stable and efficient.

The pelvis must be considered as a "pelvic vertebra" or first vertebra of the spine, as proposed by Dubousset [1]. The location of this base determines the position of the lumbar spine and hence of the entire vertebral column. In the frontal plane, the alignment is simple: The vertebral column is vertical with a median axis that passes through the middle of the sacrum.

The geometries of the pelvis and of the vertebral column in the sagittal plane are more complex. To better understand sagittal alignment, we must examine the position of the centre of gravity and the resultant line of gravity.

These two parameters have been studied by many researchers. The line of gravity is determined from force platforms (a device that represents the vertical projection of the sum of ground reaction forces, of a standing person) [2].

In the sagittal plane, this line of gravity is a line perpendicular to the ground which passes slightly behind the femoral heads in normal well-aligned subjects [2, 3]. In a 
Fig. 2 Different types of pelvises. Primate (left) and human pelvises (right) a

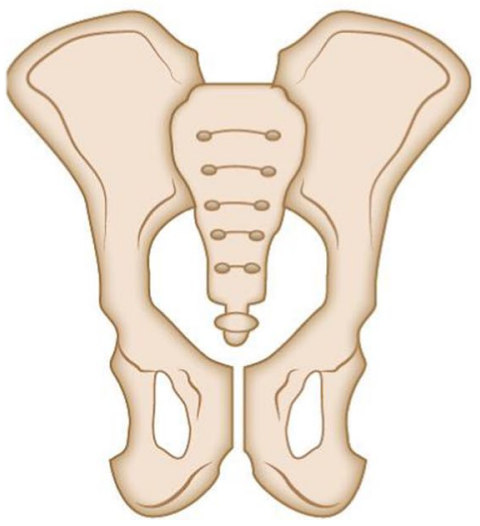

$\mathrm{PI}=\mathrm{PT}+\mathrm{SS}$

$\mathbf{a}$

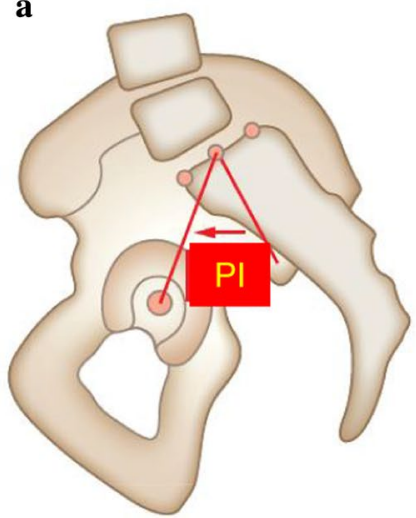

b

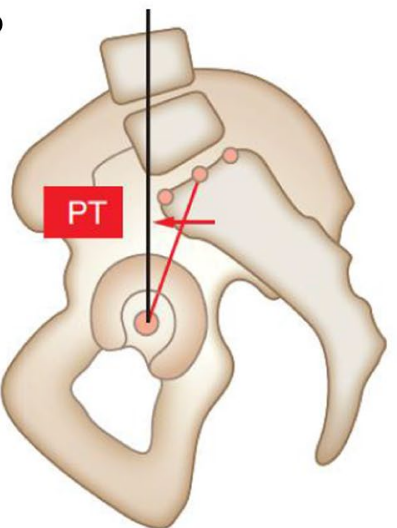

b

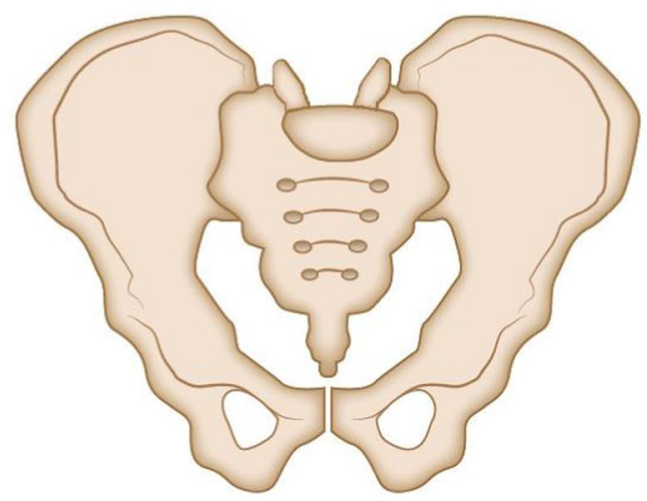

c

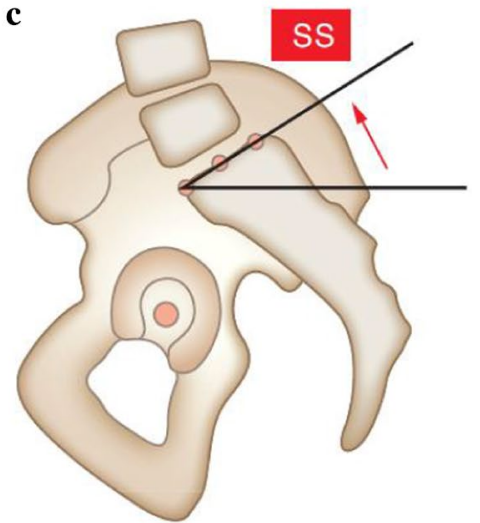

Fig. 3 Pelvic parameters. Definition of the different pelvic angles: PI angle of pelvic incidence, $P T$ pelvic tilt, $S S$ sacral slope. These last two parameters reflect the spatial orientation of the pelvis

well-aligned and balanced person, this line of gravity is projected onto the ground and delineated by the feet. The centre of gravity is located on this line in front of the S2 vertebra when the arms are alongside the body. Conventional imaging, such as magnetic resonance imaging (MRI) or computed tomography (CT), is not very useful to analyse balance because patients are lying down. It is therefore important to produce long-standing radiographs. Although the gravity line is an interesting parameter, it is not very usable because imaging devices are rarely coupled to force platforms. It is therefore essential to determine parameters that measure sagittal balance indirectly and therefore detect pathologic conditions by the radiographic analysis of standing persons keeping in mind that roughly the gravity line crosses the femoral heads on a sagittal view $[2,3]$.

\section{Definition of the pelvic parameters (Fig. 3a-c)}

The first pelvic parameter to be considered is pelvic incidence (PI). The pelvic incidence corresponds to the angle between the perpendicular to the upper S1 level passing through its centre and the line connecting this point to the axis of the femoral heads [4] as described by Legaye and Duval-beaupère [5].

It is an anatomical parameter, constant for each individual, independent of the spatial orientation of the pelvis. In fact, this angle involves the first three sacral vertebrae, the two sacroiliac joints and the posterior margin of the iliac wings up to the acetabulum.

The mobility of the sacroiliac joints is considered negligible beyond childbirth. Thus, once growth is complete, the pelvic incidence remains constant in a given individual. A small pelvic incidence angle corresponds to a narrow pelvis (small anteroposterior dimension), while a large angle indicates a broad one (large anteroposterior dimension). In elderly over 75 years old, or some specific long fusion constructs, ligament loosening can produce some degrees of variations [6-8].

The sacral slope (SS) is defined by the angle between a line tangent to the upper S1 endplate and horizontal line. A 
vertical pelvis implies a low sacral slope, while a horizontal pelvis would have a high slope. The pelvic tilt (PT) is defined by the angle between the vertical and the line connecting the centre of the sacral endplate to the axis of the femoral heads. Those two angles are positional and related to pelvis orientation. This is possible because the pelvis can rotate around the axis of the femoral heads. This movement can be forwards (anteversion, Fig. 4a) or backwards (retroversion, Fig. 4b). The pelvic tilt (PT) angle increases during retroversion of the pelvis (Fig. 4a, b).

There is a relationship between these three parameters [5] (Fig. 3): The pelvic incidence is equal to the arithmetic sum of the sacral slope and the pelvic tilt $(\mathrm{PI}=\mathrm{PT}+\mathrm{SS})$. It follows that a patient with a high pelvic incidence angle has a greater potential for pelvic retroversion. This is an important knowledge when analysing the compensatory mechanisms.

To each angle of pelvic incidence, correspond a theoretical value of sacral slope and pelvic tilt in a normal asymptomatic population. Several studies have successfully stratified these values according to the pelvic incidence value $[9,10]$. The more recent formula [9] is given by the $3 \mathrm{D}$

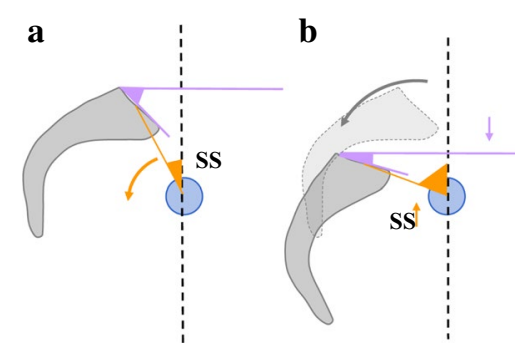

Fig. 4 a Normal pelvic version and b pelvic tilt increase with more vertical sacrum. From left to right: the angle of pelvic incidence angle remains fixed, while the pelvic tilt increases and the sacral slope declines: This corresponds to retroversion of the pelvis

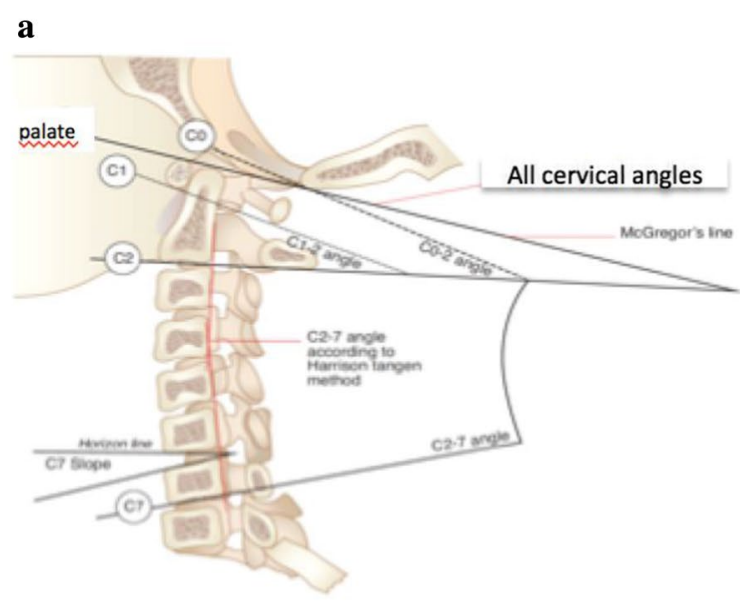

analysis of the full spine in standing position by: theoretical pelvic tilt $=0.44 \mathrm{PI}-11^{\circ}$ in a normal asymptomatic population. Therefore, it is possible to know whether the pelvic tilt measured on a X-ray taken in standing position is normal or abnormal because the pelvic tilt is a positional angle depending on the orientation of the pelvis.

\section{Analysis of spinal parameters}

There are three successive curvatures from cranial to caudal: cervical lordosis, thoracic kyphosis and lumbar lordosis [5].

Cervical parameters [11] (Fig. 5a): lower cervical shape is measured between the $\mathrm{C} 2$ endplate and the lower $\mathrm{C} 7$ endplate. The cervical spine may feature some lordosis or kyphosis, or may indeed be neutral, depending on the value of the C7 slope [11]. Cervical shape can be separated into two angles:

- High cervical angle O-C2 (occipito C2 vertebra): measurement between the McGregor line and the lower C2 endplate. The McGregor line connects the posterior margin of the bony palate to the low point of the occipital bone. This angle has an average value of $15.81^{\circ}$ $\left( \pm 7.15^{\circ}\right)$, always lordotic.

- Low cervical curvature C2-C7: measurement between the $\mathrm{C} 2$ endplate and the lower $\mathrm{C} 7$ endplate which is variable from kyphosis to lordosis in normal population.

$\mathrm{O}-\mathrm{C} 2$ and $\mathrm{C} 2-\mathrm{C} 7$ angles work inversely: When one is increasing, the other one is decreasing (Fig. 5b).

The C7 slope is a key parameter for studying the cervical spine statically [11]. The median value is $20^{\circ}$. Patients with a C7 slope greater than $20^{\circ}$ have a lordotic cervical spine (lordosis between $\mathrm{C} 2$ and $\mathrm{C} 7$ ). Patients with a $\mathrm{C} 7$ slope of less

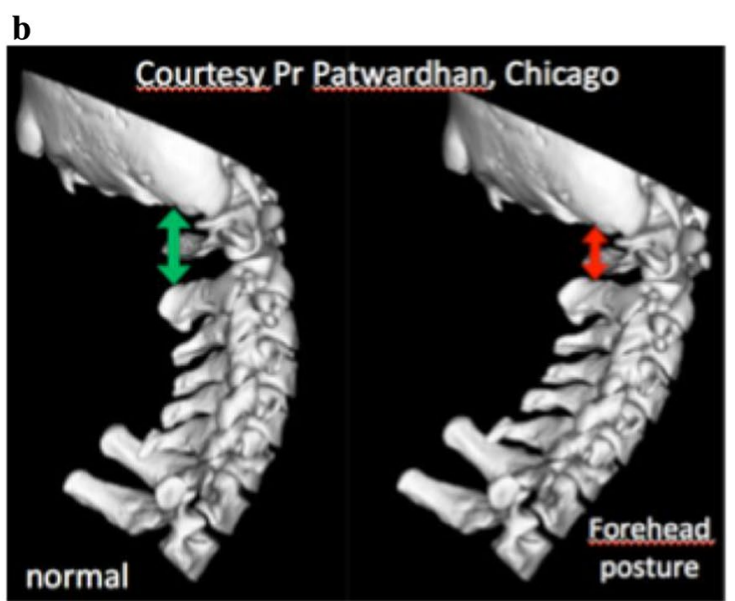

Fig. 5 a Cervical parameters: high $(\mathrm{O}-\mathrm{C} 2)$ and low $(\mathrm{C} 2-\mathrm{C} 7)$ cervical angles. b CT scan reconstruction showing the forehead posture whit increased $\mathrm{OC} 2$ lordosis and decreased $\mathrm{C} 2 \mathrm{C} 7$ lordosis 


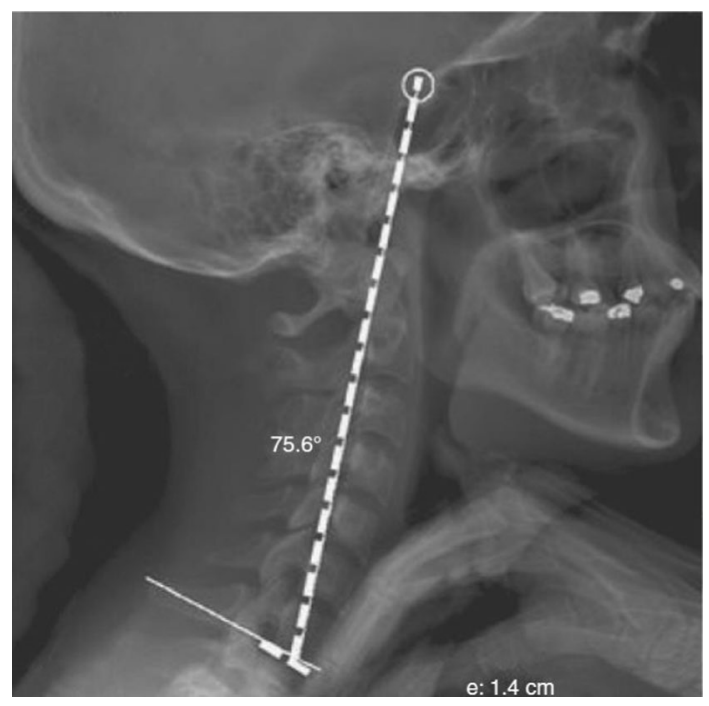

Fig. 6 Spino-cranial angle (SCA) measured on a cervical spine X-ray. The angle is measured between a line drawn from the centre of the sella turcica and a tangent to the $\mathrm{C} 7$ endplate. Cervical inclination angle (CIA) is the angle measured from centre sella turcica to each plateau of thoracic vertebrae

than $20^{\circ}$ have a neutral or kyphotic cervical spine between $\mathrm{C} 2$ and $\mathrm{C} 7$.

The spino-cranial angle (SCA) (Fig. 6) corresponds to the angle between the tangent to the upper $\mathrm{C} 7$ plateau and the line connecting the middle of the upper $\mathrm{C} 7$ plateau to the centre of the sella turcica [11]. In asymptomatic persons, the SCA value is constant with a mean of $83^{\circ} \pm 9^{\circ}$. It is an important angle because it provides an idea of the head offset over C7-T1.

The vertical cervical offset, also called cervical SVA, corresponds to the horizontal distance of the $\mathrm{C} 2$ and $\mathrm{C} 7$ plumb lines. It is a way to analyse the offset of the head.

Thoracic parameters Thoracic kyphosis is measured between the upper T1 endplate and the lower T12 endplate. It has been shown that the theoretical value of thoracic kyphosis is equal to 0.75 times the global lumbar lordosis angle, L1 to S1 [9]: T1T12 lordosis $=0.75 \times$ L1S1 lordosis.

However, many articles measure thoracic kyphosis between $\mathrm{T} 4$ and $\mathrm{T} 12$ because of the poor quality of normal radiographs due to the superposition of the humeral heads. Using EOS imaging technology [12], Le Huec and Hasegawa have recently published the value of T1-T4 kyphosis in an asymptomatic population [9]. This study shows that this segment of the kyphosis represents $8^{\circ}$ to $10^{\circ}$ of the overall thoracic kyphosis. This fact is essential because many thoraco-lumbar posterior fusion constructs stop at T4 and therefore ignore the residual lever arm of the suprajacent body segment. Analysing the position of the head and its relationship with the thoracic shape with the

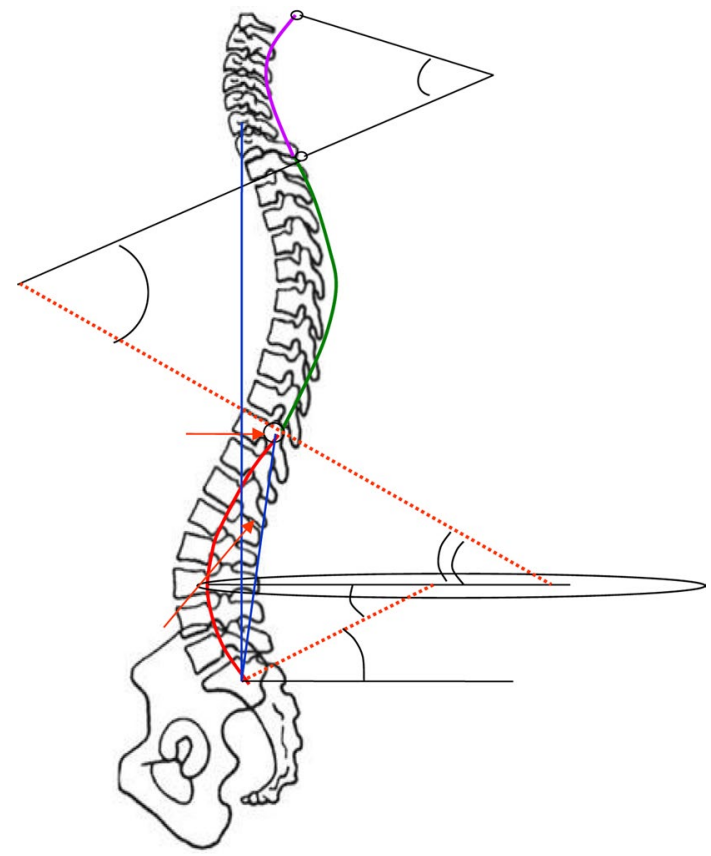

Fig. 7 Berthonnaud's biomechanical construction. Lumbar parameters: apex of the lordosis, upper and lower arch of the lumbar lordosis. The apex of the lumbar lordosis is defined as the most anterior point on a vertically positioned spine. Position of the apex varies between L3 and L5. The upper point is the inflection point that delimits the transition to thoracic kyphosis

cervical inclination angle (CIA), it has been demonstrated that the T1T5 segment is the base of support for the cervical spine with the CIA angle (a line from sella turcica to the vertebral plateau of a thoracic vertebra and a line parallel to the chosen vertebral plateau are constant in an asymptomatic population) [13].

Lumbar parameters (Fig. 7) The lumbar lordosis (LL) according to Roussouly is measured between the point of inflection from lumbar lordosis to thoracic kyphosis and the upper S1 endplate (Fig. 7) [14]. This point is geometrically calculated when the lumbar lordosis turns to the thoracic kyphosis. Using this new analysis, it is shown that twothirds of the lumbar lordosis are located at the last two lumbar levels as previously reported by Jackson [15] and confirmed by Roussouly [16]:

$\mathrm{L} 4 \mathrm{~S} 1=0.66 \times \mathrm{L} 1 \mathrm{~S} 1[16]$

A recent retrospective study suggested that this percentage of distal lordosis varies by a few degrees according to the PI value. However, the proximal lordosis seems to be significantly influenced by the PI value (greater PI, more proximal lordosis) [17]. 
Fig. 8 Different types of spine according to Roussouly. This classification allows analysis of spino-pelvic parameters in healthy persons but does not allow, in pathologic spinal balance conditions, to differentiate types 1 and 2 and therefore to plan the extent of surgical correction for these two types. It is more important for types 3 and 4. The different types also have different degenerative patterns, as shown by Barrey et al. [20]

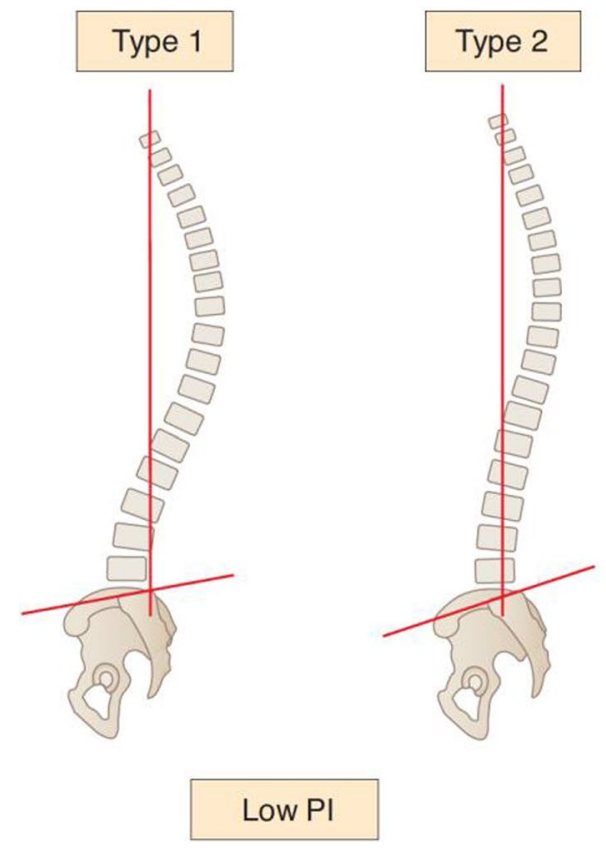

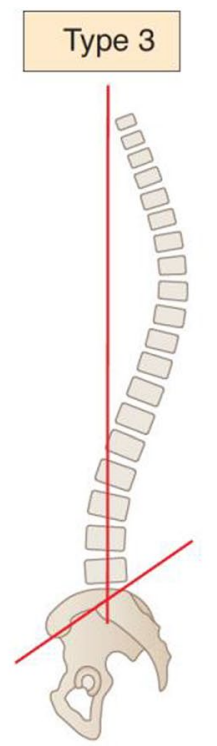

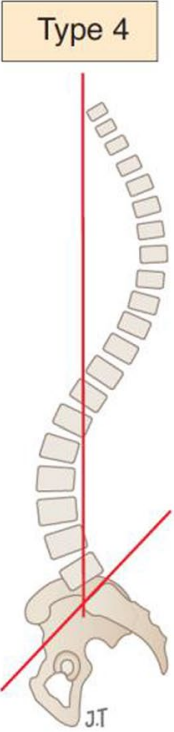

Hight PI

\section{Relationship between pelvic and spinal parameters is essential}

There is a close relationship between lumbar lordosis and pelvic incidence. Berthonnaud proposed a model that divides the lumbar lordosis into two tangential arches (Fig. 7) [18]. The lower arch is constructed by the horizontal line passing through the apex of the lordosis and by a line tangent to the sacral endplate. The upper arch corresponds to the angle measured between the horizontal line passing through the apex of the lordosis and another passing through the point of inflection. The superior arch has a constant value between $15^{\circ}$ and $19^{\circ}$ [18]. The inferior arch varies depending on the orientation of the sacral endplate and corresponds to the value of the sacral slope. There is a strong correlation between sacral slope and lumbar lordosis $(r=0.86$, $p<0.001)$. The relation between the sacral slope and the pelvic incidence explains the link between pelvic incidence and lumbar lordosis. Several studies sought to find a mathematical relationship between lumbar lordosis and pelvic incidence.

Legaye [4] demonstrated predictive formulas for lumbar lordosis as a function of pelvic parameters and vertebral rotation for scoliotic patients. These are the first predictive formulas for calculating lumbar lordosis. However, this study is limited because of the small number of patients. Schwab [19] proposed the following formula: $\mathrm{PI}=\mathrm{LL} \pm 9^{\circ}$ which again was calculated on a small number of subjects and therefore has poor accuracy for the low and high values of pelvic incidence leading to errors in daily practice.

Recently, Roussouly [10], with a large database using standard full spine and Le Huec [9], combining 3D databases using low-dose EOS imaging technology on 268 healthy adults from various ethnicities (proved by functional and pain scores: VAS, Oswestry, SF36 and SR22), has proposed a new formula for lumbar lordosis (LL):

$\mathrm{LL}(\mathrm{L} 1-\mathrm{S} 1)=0.54 \times \mathrm{PI}+27.6$

The value of this formula, calculated from a statistically powerful sample, in 3D and without possible distortion, is therefore more accurate than formulae derived from a statistically weak sample. This study also derives quite simple formulas relating the pelvic incidence to the pelvic tilt and the sacral slope:

PT $=0.44$ PI-11.4

Using those formulas allows to know the theoretical normal value of SS and PT for asymptomatic healthy young population when the PI is measured, and detect a compensating pelvic retroversion, for instance, in pathologic cases.

In a prospective study of normal asymptomatic subjects, Roussouly [10] considered these different spinal and pelvic parameters to define four types of spines (or spino-pelvic types), in particular taking into account the inflection point (corresponding to the transition biomechanical from "lumbar lordosis" to "thoracic kyphosis") (Fig. 8). This is the first classification described to stratify the population's spine shape, which is a continuum, to categories to help to understand the spine pathologies.

Types 1 and 2 are characterized by a small sacral slope (less than $35^{\circ}$ ): 
- Type 1 has a small inferior lordosis arch with a lordosis apex located further below (about L5). The lordosis is therefore "short," and the kyphosis is thoraco-lumbar.

- Type 2 has a flattened lower arch, with very little curvature. The back is "flat".

Type 3 corresponds to an average sacral slope (between $35^{\circ}$ and $45^{\circ}$ ) with a lordotic apex at L4. The lordosis is almost equally distributed over the two arches. This is the most balanced type.

Type 4 corresponds to a steep sacral slope (greater than $45^{\circ}$ ) with a lordotic apex at the anterior-inferior corner of L3. The global lordosis angle (LL) is larger and includes more vertebrae than the other types. The thoracic kyphosis is shorter.

Normative values in the asymptomatic population are very important to know to have a better analysis of the pathological situations.

Barrey et al. [20] were the first to grouped 154 asymptomatic volunteers, described pelvic and spinal parameters as a function of pelvic incidence. This study stratified the study subjects into 6 groups according to their pelvic incidence angle. However, the extreme groups did not have a sufficient number of persons to detect a statistically significant difference. The work of Le Huec and Hasegawa reinforces Barrey's study with subgroups of sufficient size [9].

From these two studies, we can conclude that overall, for pelvic incidences below the average value $\left(50^{\circ}\right)$, lordosis angle tends to increase, while the incidence decreases, whereas when pelvic incidence is around $50^{\circ}$, the lordosis tends to equal the incidence angle value $(\mathrm{LL}=\mathrm{PI})$ ), and when the incidence is over $65^{\circ}$, the lordosis tends to be less than the PI. Thus, the formula often used PI $=\mathrm{LL}+9^{\circ}$ is only valid for small incidence angles, which leads to numerous estimation errors when the pelvic incidence is greater than $50^{\circ}$ [19]. This could be one of the reasons of overcorrection of lordosis when performing posterior substraction osteotomy in patients with high incidence angle.

\section{Age-related variations of sagittal balance}

The spine balance is a dynamic phenomenon, and therefore, it is important to analyse parameter variations due to ageing process and changes during daily activities.

\section{Variations in the shape of the spine with ageing}

Physiological spinal ageing begins with the degenerative loss of disc height and thus leads to loss of lumbar lordosis, which disrupts pelvic balance. Battie showed that $74 \%$ of disc degeneration is due to genetic factors [21]. With advancing age, the lumbar spine undergoes a number of modifications. These are then associated with disc mechanical incompetence (loss of elasticity, hypermobility, etc.), hypertrophy of the articular facets, bone remodelling and atrophy of the extensor muscles. These changes lead to a lumbar hypolordosis or kyphosis which may be the cause of an anterior sagittal imbalance [20,21].

The modification of lumbar lordosis is directly related to degenerative phenomena. On the other hand, the modification of the other spino-pelvic parameters is linked to compensatory mechanisms which aim to maintain the upright position with minimal muscular effort so as to keep a horizontal gaze and the head over the pelvis.

Recently, Amabile [22] have showed that the OD-HA angle (odontoid hip axis angle), which characterizes the overall spinal balance, remains constant whatever the age and despite variations of lordosis (which decreases with loss of disc height) and the presence of compensation mechanisms.

The four spino-pelvic types described by Roussouly have also different biomechanical characteristics and, as such, do not all show the same degenerative patterns (Fig. 8). Barrey et al. [20] showed that they represent different predisposing morphotypes:

Type 1: Combines a short distal lordosis and thoracolumbar kyphosis. The sacral slope is low $\left(<35^{\circ}\right)$ as is the pelvic incidence. This type of spine shows a zone of increased mechanical compressive stress at thoracolumbar junction and a short distal lumbar hyperextension, which cannot be compensated by pelvic retroversion in those patients with low pelvic incidence. This arrangement spares lower lumbar discs but puts pressure on the distal facet joints. This type of spine is predisposed to L5S1 spondylolisthesis with isthmic lysis (nutcracker mechanism), thoraco-lumbar discopathies and degenerative junctional listhesis.

Type 2: Low sacral slope and pelvic incidence. The distribution between kyphosis and lordosis is harmonious, but the back is "flat" due to the low lordosis angle. The intervertebral discs are horizontal. There is increased stress in the L4L5 and L5S1 discs, resulting in their earlier degeneration. The potential for pelvic retroversion being very low, iatrogenic hypolordosis is very poorly tolerated.

Type 3: This is a back with harmonious lumbar and thoracic curves. There is therefore no tendency for any degeneration based on predisposing mechanical factors. Type 4: Steep sacral slope $\left(>35^{\circ}\right)$ and high pelvic incidence $\left(>55^{\circ}\right)$. The stresses are concentrated on the posterior elements of the lumbar spine. This type of back is predisposed to isthmic spondylolisthesis (traction lysis) and lumbar stenosis by posterior facet arthrosis. Note that the loss of lordosis in this type of spine is compensated over a long period due to the high pelvic 
incidence, which increases the capacity for pelvic retroversion.

\section{Part II: Sagittal balance analysis with ageing process and pathological conditions}

The study of the changes in the spine during ageing makes it possible to show compensation mechanisms at three levels: spinal, pelvic and lower limbs. Understanding these different data allows for better planning of the surgical management of the patients. It involves the creation of an image of the entire spine and the measurement of the spino-pelvic parameters to determine, by integrating all these parameters, the extent of the correction to be performed during surgery. Taking these parameters into account also enables us to understand the complications involved in this type of surgery: transitional syndromes or junctional syndromes. It is also possible to integrate these parameters into the study of gait, an area still under investigation.

\section{Compensating mechanisms in sagittal imbalance}

To adapt to the variations in the shape of the spine, several compensation mechanisms are implemented at the segmental, regional and global levels (Fig. 9) [23]. These mechanisms are not all implemented at the same time but are associated with different extents.

The basic concept of spinal compensation is the extension of the adjacent spinal segments in order to avoid the anterior translation of the gravity line due to progressive disc degeneration and loss of disc height. This, however, causes adverse effects.

\section{At the segmental level}

There is frequently hyperextension of adjacent segments at the lumbar level: This hyperextension can be mono- or multi-segmental and is defined by a single segmental lordosis above $15^{\circ}$. This mechanism causes an increase in posterior stresses and results in retrolisthesis, facet hypertrophy and excessive pressure on the spinous processes (Baastrup syndrome). This also can reduce the diameter of the intervertebral foramen and central canal. An indicator worth noting is the "leaning back sign" described by Faundez et al. [24] and characterized by a hyperextended disc with anterolisthesis at the same level: The upper vertebra is slipped forward and leaning in hyperextension on the vertebra below (Fig. 10).

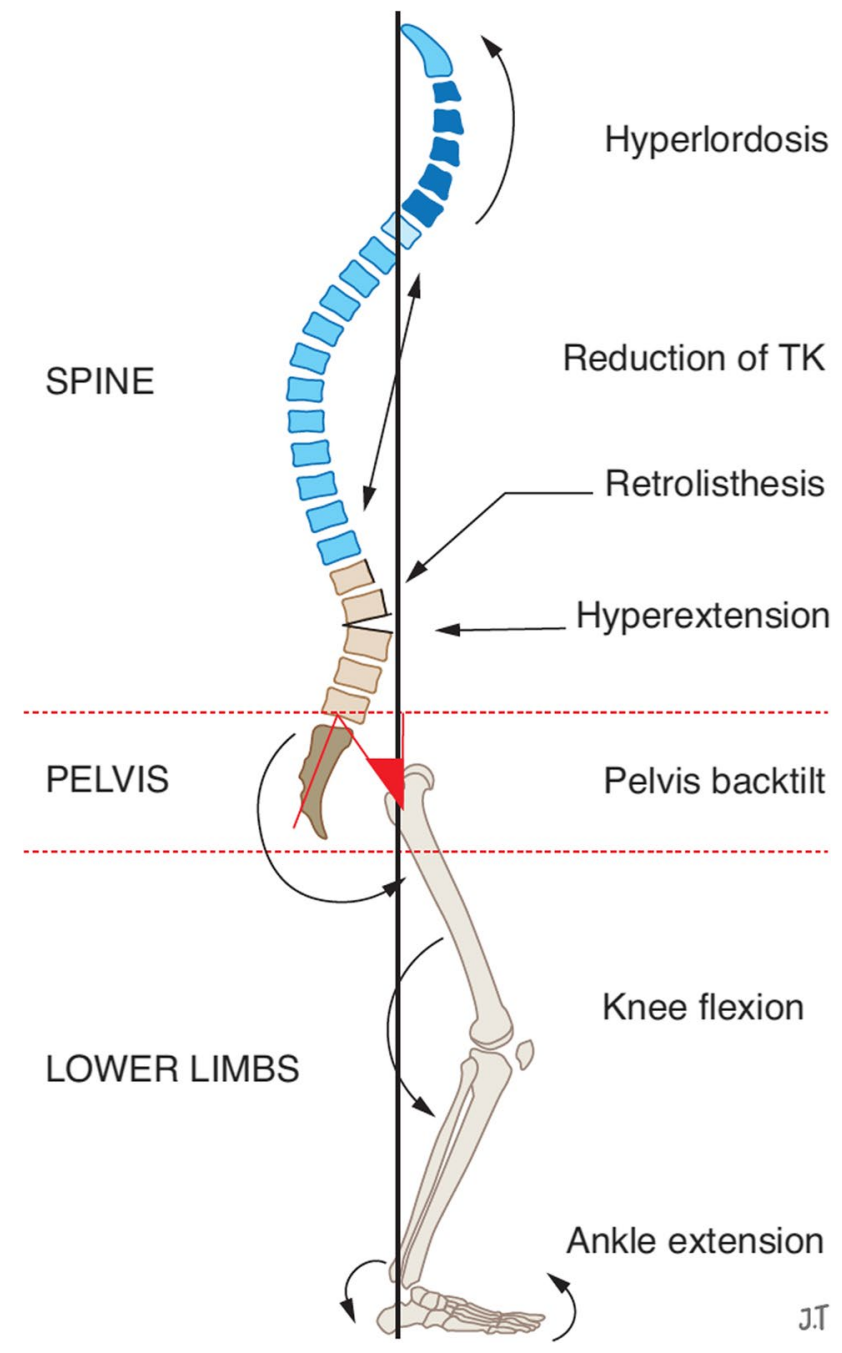

Fig. 9 Compensation mechanisms for anterior segment imbalance. Cervical hyperlordosis, reduction in thoracic kyphosis, lumbar retrolisthesis, hyperextension of the lumbar segments, pelvic retroversion, bending of the knees and stretching of the ankles

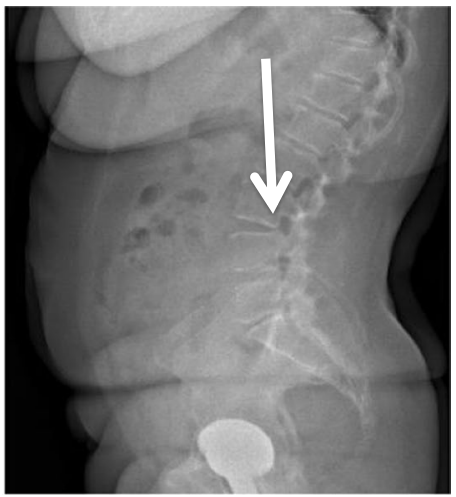

Fig. 10 a Leaning back sign drawing. b X-ray of a patient with a leaning back sign, which is a vertebra leaning by its posteroinferior angle, on the endplate below, and in segmental hyperextension. It represents a compensating mechanism of high severity on a mobile disc 


\section{At the regional level}

The sole mechanism at the level of the pelvic is retroversion. It corresponds to a posterior rotation of the pelvis around the femoral heads, translated in an increase in pelvic tilt and a decrease in sacral slope. Pelvic retroversion translates into extension of the hip joints. Several studies have shown that chronic low back pain patients have a retroverted pelvis [15]. The higher the value of the pelvic incidence, the greater the retroversion can be. This is caused by contraction of the extensor muscles of the hips (gluteal muscles) [25, 26]. The range of hip extension between the natural upright position (normal population) and that of maximum extension (pelvic retroversion) can be considered as the "extension reserve" A well-functioning spine requires this hip extension, allowing it to assist in the compensation of sagittal imbalance $[27,28]$. Hovorka has proposed methods of radiographic measurement of this hip extension reserve [27]. Lazennec also proposed a measurement using the EOS imaging system [28]. The hip extension reserve is included in the pelvic retroversion capacity. This represents only few degrees, but those are important to know as this possibility is missing in case of hip arthrosis leading sometimes to limited retroversion in case of hip flexum [27].

Another regional compensation mechanism is knee flexion, which usually happens after maximal pelvic retroversion has been reached.

\section{At the thoracic and cervical level}

The decrease in thoracic kyphosis usually observed in younger subjects because it results from the contraction of erector muscles, which are still strong and efficient.

Cervical hyperlordosis also results from the contraction of erectors muscles in the cervical spine and can eventually produce facet joint hyperpressure, arthritis with foraminal or central stenosis (myelopathy). The forehead posture is an important compensatory positioning of the cervical spine allowing to keep the cervical foramen size open and allowing to keep the gaze horizontal by limiting cervical lordosis using the $\mathrm{O}-\mathrm{C} 2$ hyperlordosis as a compensation (Figure 5 b). E Ferrero [29] showed also that the cranial sagittal vertical axis (Cr SVA) is a better radiographic measure to predict clinical outcomes in adult spinal deformity surgery than the C7 SVA.

In the lower limbs Knee flexion and ankle stretching also help in shifting the line of gravity backwards. Knee flexion has been shown to be correlated with loss of lumbar lordosis [22]. Considering the feet as the base of support, one compensatory mechanism to keep the head and the gravity line in good position is the concept of pelvis translation as shown by Ferrero [29].

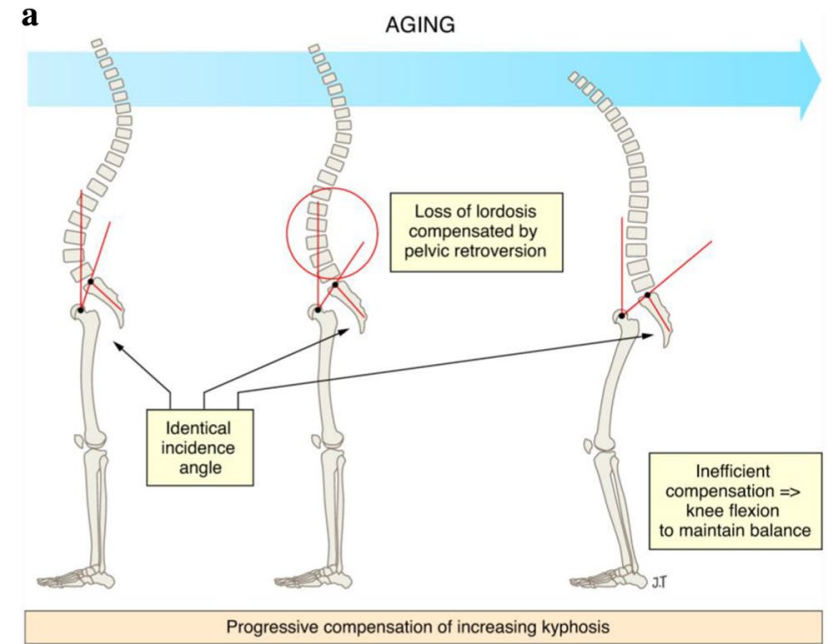

b

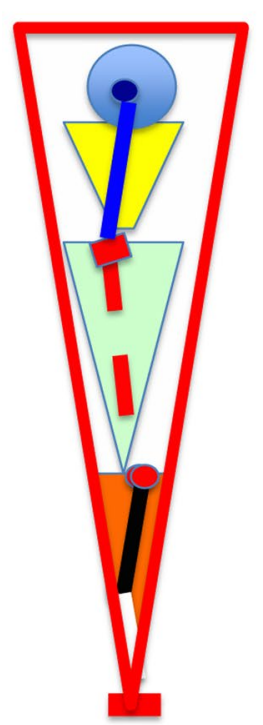

Fig. 11 Compensation mechanisms for age-related imbalance. a This figure depicts from left to right, the same person at a young age (left) then at older ages (right). With ageing, lumbar lordosis decreases and different compensating mechanisms act consecutively. These are shown from left to right. b The 3 conus concept integrating the full body balance: The lower conus includes the lower limb and the femoral head. The median conus includes thepelvis and the thoracolumbar spine up to $\mathrm{T} 1$ and the upper conus includes the cervical spine and the head

All these mechanisms combine in different ways depending on the person. This compensation is of limited efficiency, especially in elderly persons, who have a weakened musculature and often arthritic joints (Fig. 11). To integrate all those compensatory mechanisms in a new balance concept to complete the Dubousset's conus of economy, we proposed to use the new 3 conus concepts in order to split the analysis at each level and have a better analysis of the global balance. The lower conus includes the lower limb and the femoral head. The median conus includes the pelvis and the 
thoraco-lumbar spine up to T1, and the upper conus includes the cervical spine and the head. Each conus can adapt its compensatory mechanisms but always in a way to keep the head over the 2 feet base of support [30] (Fig. 11 bis).

\section{Overall assessment of patients with spinal imbalance}

How do we integrate these data before treating those patients or during surgical planning before spinal surgery? The goal is to move in two steps:

- Determine the value of the pelvic incidence and therefore determine the theoretical values of the spino-pelvic parameters.

- Analyse the overall sagittal balance from cervical spine to lower limbs

Several indicators have therefore been defined so as to evaluate the overall spinal balance. All this can be evaluated only by obtaining full spine radiographs on large cassettes or with the EOS low-dose imaging system (EOS imaging, Paris, France) [12].

Radiography of the entire spine is mandatory. The analysis of sagittal balance requires radiographic anteroposterior and lateral views of the entire spine in standing and relaxed positions, from $\mathrm{C} 2$ to the femoral heads. The position must also be standardized: hands resting on collarbones [31]. These radiographs can be made on large cassettes or with the EOS imaging system. The EOS imaging system enables us to produce images of the entire spine while considerably reducing the radiation dose (8-10 times less than standard radiology) and much faster than with traditional imaging systems [32]. The image capture is very fast, allowing also the 3D modelling. This system avoids the phenomena of vertical distortion found in large cassette imaging.

Overall assessment of balance can be performed using different parameters:

\section{The C7 plumb line [15, 33] (Fig. 12):}

This is the lowered vertical line of C7. Ideally it must pass through the sacral endplate, but even if this is the case, it does not imply that the spino-pelvic parameters are adequate: The spine can be in (compensated) balance, but spino-pelvic parameters can be inadequate (not aligned).

The sagittal vertical axis [15, 33] (Fig. 13): Jackson also described the sagittal vertical axis or SVA which corresponds to the horizontal distance between the $\mathrm{C} 7$ plumb line and the posterior-superior S1 corner.

The SVA is correlated with quality-of-life parameters [34]. The normal SVA should be less than $5 \mathrm{~cm}$, but this parameter is age-dependent [35]. However, in our opinion,
Fig. $12 \mathrm{C} 7$ plumb line and gravity line are not similar

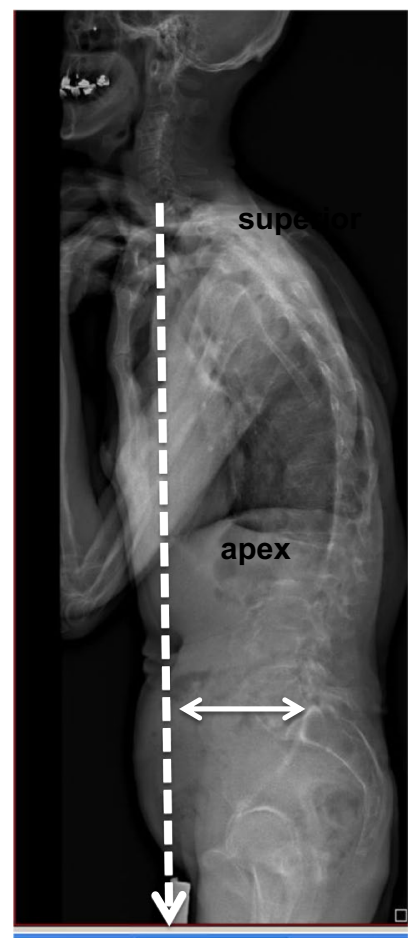

Fig. 13 Sagittal vertical axis: SVA is a good parameter to analyse the balance of the same person over time, but not to compare patients balance between themselves 
$\mathbf{a}$

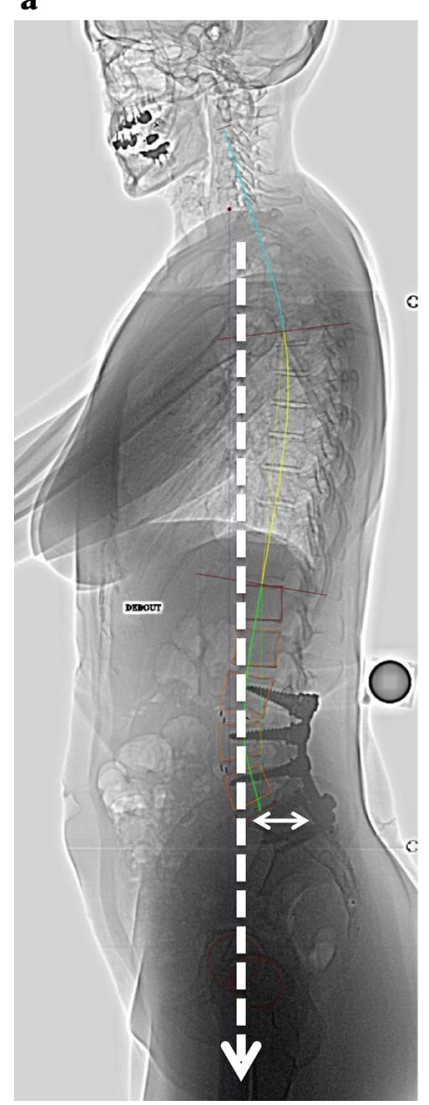

b

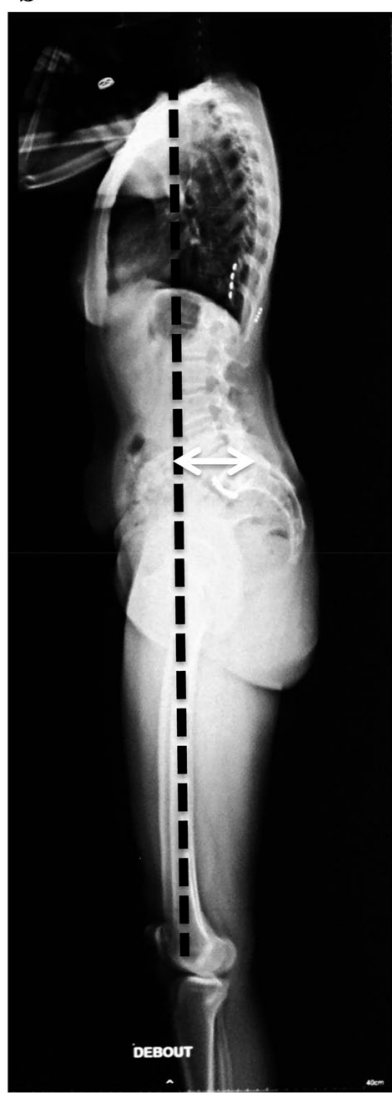

Fig. 14 a Small SVA: 4, $5 \mathrm{~cm}$ (in normal range) but imbalanced spine due to small pelvic incidence with limited pelvic retroversion: Patient is leaning forward. SVA is not a good marker of balance. b High SVA more than $5 \mathrm{~cm}$ but well-balanced patient because of large pelvic incidence

it does not take into account the value of pelvic incidence, which induces considerable interpretation bias, particularly in high pelvic incidences. (The pelvis has a larger anteroposterior diameter in high pelvic incidence.) On the other hand, the average values are not very selective and only the subgroup stratification allows for an appropriate analysis of the balance. This parameter should be used only to compare the balance situation of a patient over time or before and after surgical treatment. It cannot be used to compare the results of a series of patients because their pelvic incidence angles are not the same, due to anatomical differences as explained above [20] (Fig. 14a, b)

The SVA corresponds to a measurement of distance, so it requires calibrated images as opposed to indicators that use angles.

Spino-sacral angle or SSA [20] (Fig. 15) is defined by the angle connecting the centre of the $\mathrm{C} 7$ vertebra to the centre of the $\mathrm{S} 1$ endplate and the line parallel to the superior $\mathrm{S} 1$ endplate. Its normal value is $135^{\circ} \pm 8^{\circ}$. This is an intrinsic parameter of balance [36]. It is a parameter of overall

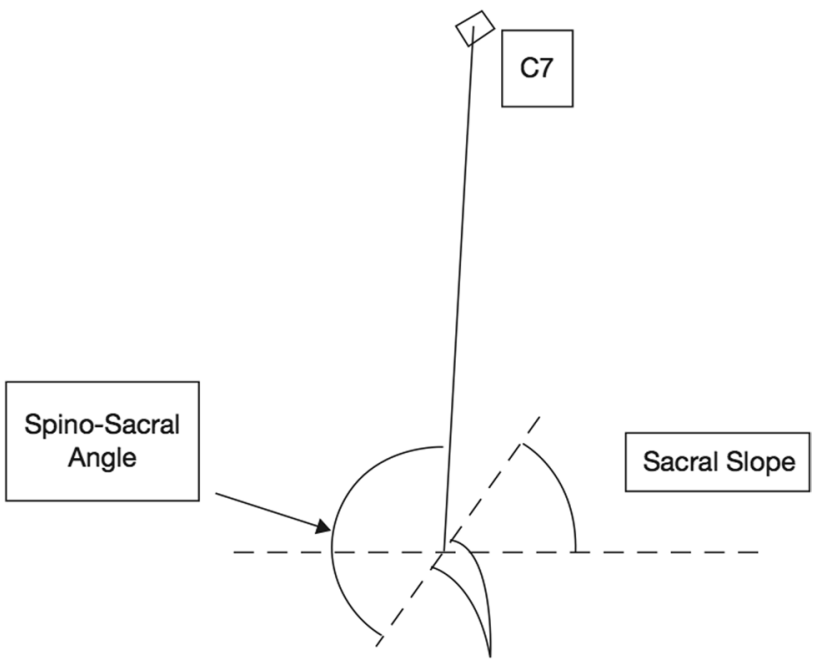

Fig. 15 Spino-sacral angle

balance because it integrates the $C 7$ position with a pelvic parameter: the sacral slope. It does not integrate the cervical spine and head.

\section{C7 Plumb line/sacrofemoral distance ratio, C7/SFD ratio [23] (Fig. 15)}

The purpose of the C7/SFD ratio (Barrey index) is to replace a distance parameter like the SVA by a ratio applicable to all radiographs and also take into account the position of the femoral head and therefore some of the thickness of the pelvis, which varies according to the angle of pelvic incidence [23].

This ratio is equal to 0 when the $\mathrm{C} 7$ plumb line is projected exactly at the posterosuperior corner of the sacrum, and is equal to 1 when it is projected on the bi-coxo-femoral axis. It is greater than 1 when the $\mathrm{C} 7$ plumb line projects in front of the femoral heads and negative when the latter projects behind the sacrum (normal value is $-0.9 \pm 1$ ). It does not integrate the cervical spine and head (Fig. 16).

\section{T1 pelvic angle, TPA [37] (Fig. 17)}

This corresponds to the angle between a line connecting the centre of $\mathrm{Tl}$ to the centre of the femoral heads and the line to the centre of the S1 endplate. It has been correlated with pelvic tilt and SVA, but does not account for pelvic incidence value. The TPA target value is $<14^{\circ}$

This is an angle somewhat similar to the SSA for thoracic-lumbar-pelvic analysis. It does not integrate the cervical spine and head.

Global tilt angle (GT) [38] described to analyse the balance takes also $\mathrm{C} 7$ as a landmark. It does not integrate the 
Fig. 16 C7/SFD ratio. This is called the Barrey index: the ratio of distance taking in account the vertical line through the femoral head and the $\mathrm{C} 7$ plumb line and a vertical line through posterior corner of S1 (normal value is -0.9 )

Fig. 17 TPA measurement (T1 pelvic angle)

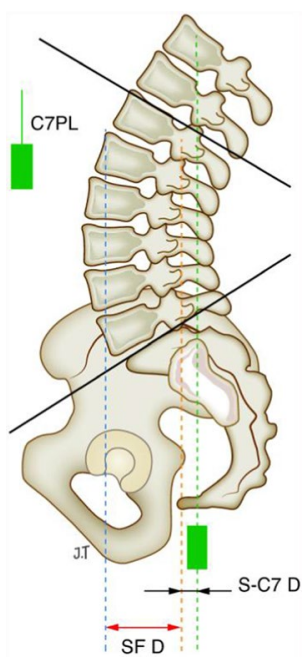

Fig. 18 FBI (Full Balance

Integrated)

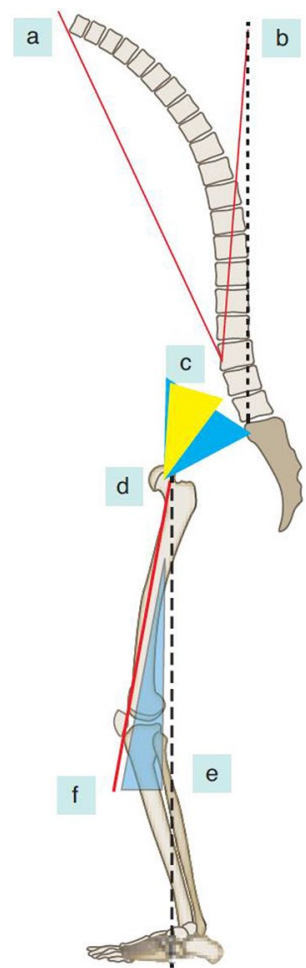

of the posterosuperior S1 corner and the apex of the angle is $L 4$ vertebra. In the normal population, the apex of the lumbar lordosis is located mostly around L4. These three points (centre of C7, "ideal" C7 and L4) form a triangle whose apex is the L4 vertebra.

- The second angle is the femoral obliqueness angle (FOA): In healthy persons, the femurs are vertical. During mechanical compensation, the knees are flexed and the femurs become oblique on a full spine lateral X-ray view. The FOA corresponds to the angle between the axis of the femoral shaft and the vertical. This angle must be added to the correction to restore a proper sagittal balance.

- The third angle is the pelvic compensation angle (PTCA): It corresponds to the difference between the theoretical pelvic tilt and the measured tilt. This compensation PTCA is equal to measured PT - theoretical value of PT $\left(\mathrm{PT}\right.$ th $\left.=0.44 \mathrm{PI}-11 \cdot 4^{\circ}\right)$. Experience has proved that if measured pelvic tilt is less than $25^{\circ}$ then PTCA is around $5^{\circ}$ and must be added to the correction, and if PTCA is greater than $25^{\circ}$ then $10^{\circ}$ must be added. This is an approximation, but the real value can be calculated as explained above.

The sum of these three measurements gives the value of sagittal angle correction required to restore a balance adapted to the patient. However, this technique can also 
$\mathbf{a}$

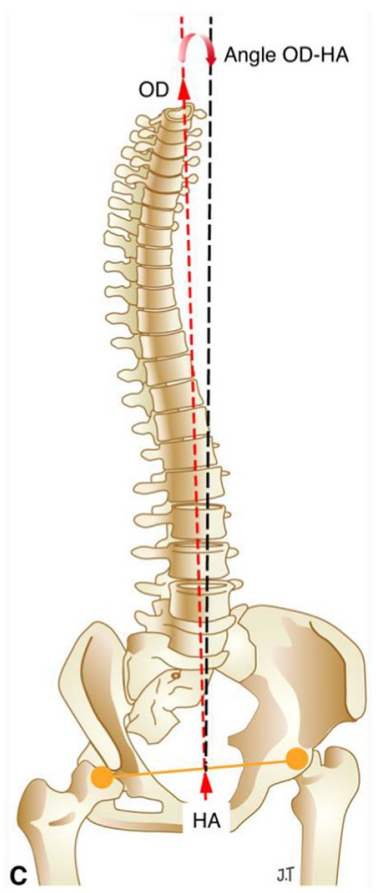

Fig. 19 a and b OD-HA measurement (odontoid hip axis). a 3D drawing of the OD-HA angle from dens of $\mathrm{C} 2$ to hip axis and vertical line through hip axis. b OD-HA measured on a 2D X-ray, lateral view. It informs on the efficiency of compensating mechanisms, as its value is normally $+2^{\circ}$ to $-5^{\circ}$ degrees in asymptomatic patients, independently of age, global spine shape and the presence or absence of compensating mechanisms

be used to analyse the overall balance. Its average value in asymptomatic people is less than $5^{\circ}[39]$ and could be negative. It does not integrate the cervical spine and head.

The OD-HA (odontoid hip axis) [40] (Fig. 19a, b): This is the angle between the vertical and the highest point of the odontoid process (dens) connecting to the centre of the acetabulum (bi-coxo-femoral axis). This angle was studied in an asymptomatic patients subgroup (SD: $1,6^{\circ}$ ). It hardly varies and is a good way to study the overall sagittal balance. It integrates the cervical spine and head and stays constant even in elderly if they are asymptomatic.

This angle takes into account the position of the cervical spine, the thoraco-lumbar spine and pelvis, and may benefit an overall analysis and assessment of the risk of proximal junctional kyphosis (PJK) after an extended thoraco-lumbar fusion. In fact, if OD-HA is positive $\left(>+2^{\circ}\right)$, above the normal value $\left(+2^{\circ}\right.$ to $\left.-5^{\circ}\right)$, it means that the patient is out of balance, and there is an increase in the lever arm on the upper instrumented vertebra (UIV), as shown in the study by Faundez et al. [13].

Preoperative planning is today mandatory before any kind of spine fusion to avoid overstress on adjacent levels as proposed by Berjano [41]

\section{Junctional syndromes adjacent to an arthrodesis}

The junctional syndrome or proximal junctional kyphosis (PJK) is defined historically by a $10^{\circ}$ increase in proximal junctional kyphosis compared to the preoperative radiograph [42]. This is measured between the inferior endplate of the first instrumented vertebra and the superior endplate of the second vertebra above it. This is the major complication in deformity correction surgery. Proximal junctional kyphosis often occurs early (within 3 months), in postoperative patients. There are risk factors which the surgeon can modify during surgery:

- Thoracoplasty [43]

- Combined methods that create an extremely rigid area [43]

- Interspinous ligament injury in the non-instrumented area [44]

- Fusion that includes the sacrum [45]

- Thoracic kyphosis exceeding the lumbar lordosis

- Arthrodesis up to T1-T3

- A notable change in SVA

- A greater than $30^{\circ}$ increase in lumbar lordosis

- An overall increase in spinal curvatures

- There are also risk factors that are not modifiable by the surgeon $[45,46]$ :

- Osteopenia

- High BMI

- Thoracic kyphosis exceeding $40^{\circ}$

- High pelvic incidence

- High preoperative SVA is not a marker to use as it should be analysed with the pelvic incidence

Several studies have shown the increase in mobility in the segment adjacent to the arthrodesis, which could explain its degeneration [47]. It is crucial to integrate the cervical spine and the head when analysing the PJK or PJF risk. The recently published CIA [13] (cervical inclination angle) (Fig. 5a) could provide a good help to predict the risk of junctional breakdown if its value is maintained around $85^{\circ}$ for the first thoracic vertebrae in long construct up to T3 or T4.

Junctional breakdowns leading to PJK and subsequently to PJF are always worsened by a too important lever arm at the superior part of the body, located above the last instrumented vertebra. Therefore, it is mandatory to be able to evaluate this lever arm preoperatively by pre-op planning as proposed recently by Faundez [13] using software allowing to simulate the post-op correction and simultaneously 


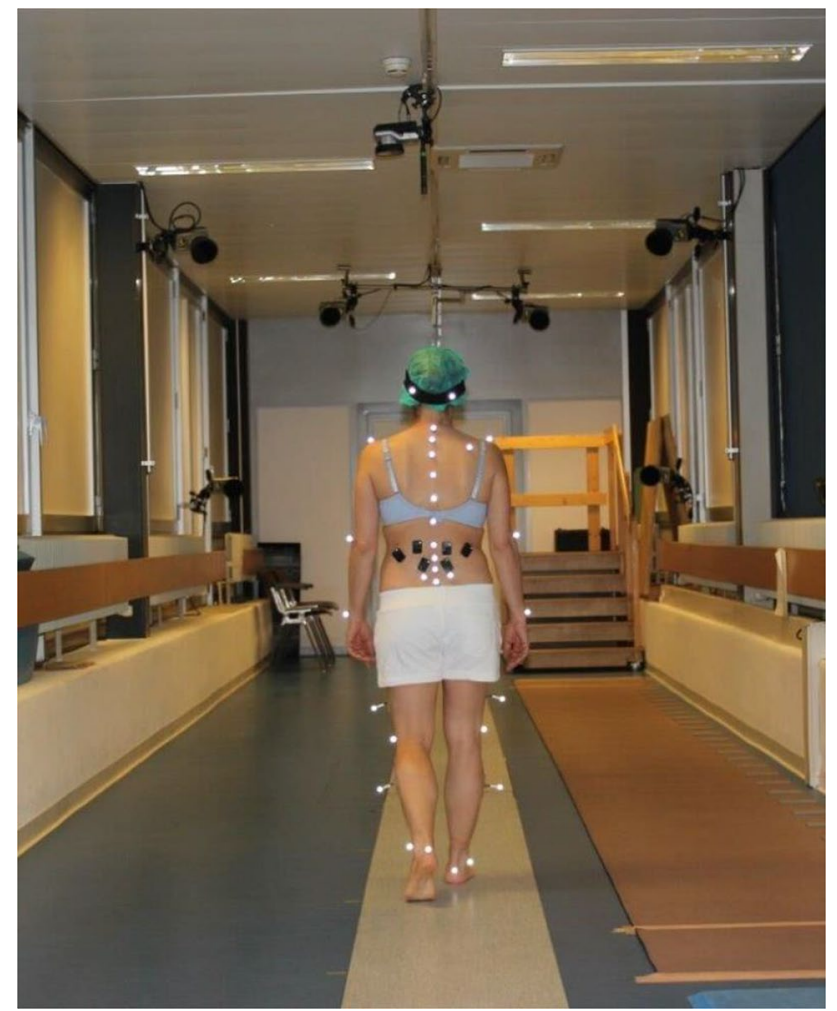

Fig. 20 Y Shiba's study, increase in imbalance during gait. On the treadmill: patient leans forward more and more over time when walking with problem of balance

with a second software to evaluate the lever arm and constraint applied at the upper instrumented vertebra (UIV). This way the construct can be longer or shorter according to the correction need and respecting the biomechanics to avoid excessive load at the UIV. This new way of thinking must be combined with preoperative control of spine correction during the surgery to be sure that the pre-op planning is fully respected during the operative procedure.

\section{Balance analysis while walking: the final stage undergoing validation}

It is also important to study the overall sagittal balance during gait in pathologic conditions. Indeed, a balanced but compensated spine, on static radiographs, can become unbalanced when set in motion. Recently, Y Shiba has performed a three-dimensional gait analysis in patients with degenerative lumbar kyphoscoliosis [48]. He demonstrated that the loss of overall sagittal alignment was underestimated on static radiographs. Indeed, the anterior imbalance occurs, or increases, as soon as a step is made and progresses along that step (Fig. 20).

This is explained by the compensation phenomena incurring in an anterior imbalance. In fact, one of the first compensatory phenomena occurs at the level of the pelvis when the extensor hip muscles, which take part in pelvic retroversion, are activated. During gait, the hip extensor muscles does alternatively contract and no longer contributes to a static pelvic retroversion. This results in pelvic anteversion. The anterior imbalance is then no longer compensated and increases from the onset of the gait. The tendency to lean forward during gait is accentuated when the posterior spinal muscles have atrophied and no longer allow the trunk to be held vertically, a common condition in patients with degenerative kyphoscoliosis.

Lee, in his study of "flat" degenerative spines, separated two groups of patients according to their pelvic tilt, during gait [49]. The first group of patients maintained pelvic retroversion during gait, while the second group could not. These findings indicated that patients who could maintain pelvic retroversion during gait (approximately $80 \%$ ) would be improved by surgical correction, unlike those who displayed dynamic pelvic anteversion.

Bae has recently proposed to make radiographs of the entire spine after a 10-min walk, so as to detect a major anterior imbalance that can or cannot be compensated on static radiographs [50].

\section{Clinical application}

These different concepts of sagittal balance allow for the analysis of the clinical condition of a patient. There are 3 types of profiles:

- balanced

- balanced but compensated

- non-compensated meaning imbalanced

This analysis suggests a therapeutic strategy. In practice, it is important to know how to treat the pathology by adapting a strategy to each individual patient: taking into account certain general problems (cardiac, pulmonary, diabetic, obesity) and the patient's ability to compensate. By allowing for a compensated balance, a simpler surgery is recommended, rather than major surgery with a high risk of complications [51]. Understanding sagittal balance provides the most appropriate choice [52]. Sagittal balance is a dynamic phenomenon, and it is better to have a good sagittal balance (even compensated) than a good sagittal alignment with a long fusion and no ability to compensate $[39,53]$. This is the reason why we came to the conclusion that the recent papers providing rules to tolerate increased pelvic tilt and SVA in elderly is probably not appropriate. The only good way to prepare a spinal fusion surgery is to make a plan and simulate what will happen after a short or long fusion. Knowing all the parameters presented above 


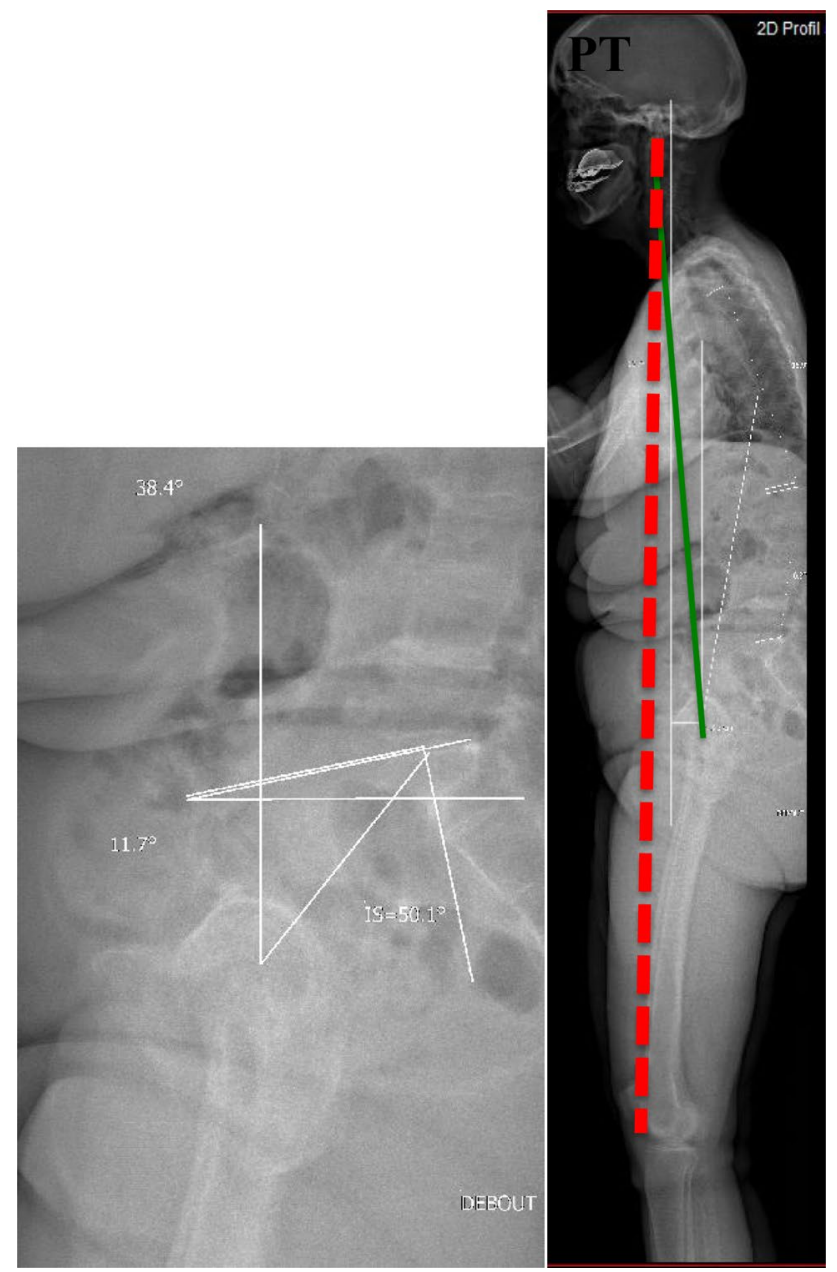

Fig. 21 Example of sagittal balance analysis. Global balance is not good: $\mathrm{C} 2-\mathrm{HA}=17^{\circ}$ (normal is: $+2^{\circ}$ to $-5^{\circ}$ ). Pelvis balance pelvic incidence at $56.1^{\circ}$. The pelvic compensation mechanisms involve a major retroversion (pelvic tilt angle at $38.4^{\circ}$ ). The sacral slope is greatly decreased by this retroversion. Thoraco-lumbar balance Radiographic analysis shows a total loss of lumbar lordosis: lordosis measured $-4^{\circ}$. Theoretical lordosis according to Le Huec's formula: $0.54 \times 56.1^{\circ}+27.6=52.4^{\circ}$. Thoracic kyphosis $\left(28^{\circ}\right)$ is less than expected (theoretically around $45^{\circ}$ for this patient), reduction in kyphosis due to posterior muscle contraction to compensate the loss of lumbar lordosis. Lower limbs: moderate knee flexion. Operative plan is to restore good L4S1 lordosis $\left(32^{\circ}\right)$, good T12 L4 lordosis around $20^{\circ}$, and this will automatically restore a normal PT and economical thoracic khyphosis as the CIA is normal $\left(85^{\circ}\right)$ in the cervical head complex

and understanding the compensatory mechanisms that will allow for a good global balance, we suggest that using the OD-HA angle would be the best way to predict the risk of failure or junctional breakdown. The muscle function can be approximately evaluated on dynamic flexion/extension $\mathrm{X}$-rays, in standing position and gait analysis after 10-min walking.

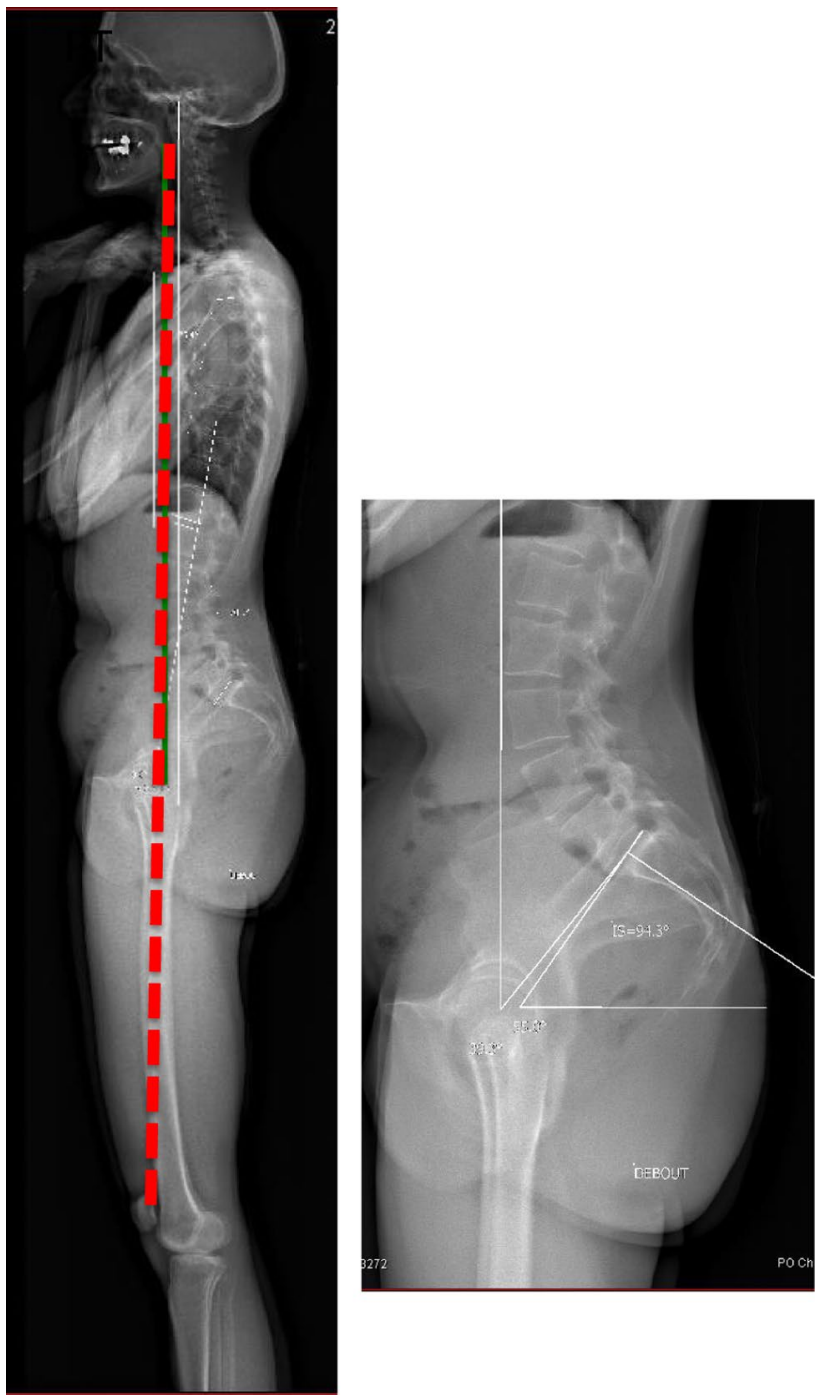

Fig. 22 Example of good sagittal balance with high pelvic incidence. Global balance is good: $\mathrm{C} 2-\mathrm{HA}=0^{\circ}$ (normal is: $+2^{\circ}$ to $-5^{\circ}$ ). Pelvis balance pelvic incidence at $94.3^{\circ}$ pelvic tilt angle at $39.3^{\circ}$ which is normal for the pelvic incidence (cf Le Huec's formula). Thoracolumbar balance Radiographic analysis shows normal lumbar lordosis $\left(74^{\circ}\right)$ and thoracic kyphosis $\left(50^{\circ}\right)$. Theoretical lordosis according to Le Huec's formula: $0.54 \times 94.3^{\circ}+27.6=78.5^{\circ}$. Lower limbs straight. In case of surgical decompression at one level, it is very important to respect this balance

Two examples of sagittal balance analysis (Figs. 21 and 22) show how to analyse the balance before any surgical treatment.

\section{Conclusion}

Sagittal balance of the spine is a vast subject which must be apprehended with the patient standing at rest in a standardized position, but also during walk, from which we can detect very harmful stresses on the osteoligamentous 
structures. The fundamental principles have been analysed on static standing radiographs, but in the future the study of gait will add fundamental information. Analysis of spinopelvic parameters helps to guide the surgical strategy in spinal surgery. It is very useful in preoperative planning, but allows us also to understand what went biomechanically wrong after a surgery.

The global analysis including the cervical spine and head is nowadays mandatory. Sagittal balance must be carefully considered before any surgery, limited or not, and especially at the lumbar level and, above all, if the L4-L5-S1 levels (which represent $66 \%$ of the lordosis) are included in the fusion.

\section{Compliance with ethical standards}

Conflict of interest The authors declare that they have no conflict of interest.

Open Access This article is distributed under the terms of the Creative Commons Attribution 4.0 International License (http://creativeco mmons.org/licenses/by/4.0/), which permits unrestricted use, distribution, and reproduction in any medium, provided you give appropriate credit to the original author(s) and the source, provide a link to the Creative Commons license, and indicate if changes were made.

\section{References}

1. Dubousset J (1994) Three-dimensional analysis of the scoliotic deformity. In: Weinstein S (ed) The pediatric spine: principles and practice. Raven Press, New York, pp 479-496

2. Roussouly P, Gollogly S, Noseda O et al (2006) The vertical projection of the sum of the ground reactive forces of a standing patient is not the same as the $\mathrm{C} 7$ plumb line: a radiographic study of the sagittal alignment of 153 asymptomatic volunteers. Spine 31:E320-E325. https://doi.org/10.1097/01.brs.0000218263.58642 .ff

3. Schwab F, Lafage V, Boyce R et al (2006) Gravity line analysis in adult volunteers: age-related correlation with spinal parameters, pelvic parameters, and foot position. Spine 31:E959-E967. https ://doi.org/10.1097/01.brs.0000248126.96737.0f

4. Legaye J, Duval-Beaupère G, Hecquet J, Marty C (1998) Pelvic incidence: a fundamental pelvic parameter for three-dimensional regulation of spinal sagittal curves. Eur Spine J Off Publ Eur Spine Soc Eur Spinal Deform Soc Eur Sect Cerv Spine Res Soc 7:99-103

5. Duval-Beaupère G, Schmidt C, Cosson P (1992) A Barycentremetric study of the sagittal shape of spine and pelvis: the conditions required for an economic standing position. Ann Biomed Eng 20:451-462

6. Bao H, Liabaud B, Varghese J et al (2018) Lumbosacral stress and age may contribute to increased pelvic incidence: an analysis of 1625 adults. Eur Spine J Off Publ Eur Spine Soc Eur Spinal Deform Soc Eur Sect Cerv Spine Res Soc 27:482-488. https://doi. org/10.1007/s00586-017-5324-z

7. Jean L (2014) Influence of age and sagittal balance of the spine on the value of the pelvic incidence. Eur Spine J Off Publ Eur Spine Soc Eur Spinal Deform Soc Eur Sect Cerv Spine Res Soc 23:1394-1399. https://doi.org/10.1007/s00586-014-3207-0
8. Cecchinato R, Redaelli A, Martini C et al (2017) Long fusions to $\mathrm{S} 1$ with or without pelvic fixation can induce relevant acute variations in pelvic incidence: a retrospective cohort study of adult spine deformity surgery. Eur Spine J Off Publ Eur Spine Soc Eur Spinal Deform Soc Eur Sect Cerv Spine Res Soc 26:436-441. https://doi.org/10.1007/s00586-017-5154-z

9. Le Huec JC, Hasegawa K (2016) Normative values for the spine shape parameters using 3D standing analysis from a database of 268 asymptomatic Caucasian and Japanese subjects. Eur Spine J Off Publ Eur Spine Soc Eur Spinal Deform Soc Eur Sect Cerv Spine Res Soc 25:3630-3637. https://doi.org/10.1007/s0058 6-016-4485-5

10. Roussouly P, Gollogly S, Berthonnaud E, Dimnet J (2005) Classification of the normal variation in the sagittal alignment of the human lumbar spine and pelvis in the standing position. Spine 30:346-353

11. Le Huec JC, Demezon H, Aunoble S (2015) Sagittal parameters of global cervical balance using EOS imaging: normative values from a prospective cohort of asymptomatic volunteers. Eur Spine J Off Publ Eur Spine Soc Eur Spinal Deform Soc Eur Sect Cerv Spine Res Soc 24:63-71. https://doi.org/10.1007/s0058 6-014-3632-0

12. Dubousset J, Charpak G, Skalli W et al (2008) Skeletal and spinal imaging with EOS system. Arch Pediatr Organe Off Soc Francaise Pediatr 15:665-666. https://doi.org/10.1016/S0929 $-693 X(08) 71868-2$

13. Faundez AA, Richards J, Maxy P et al (2018) The mechanism in junctional failure of thoraco-lumbar fusions. Part II: analysis of a series of PJK after thoraco-lumbar fusion to determine parameters allowing to predict the risk of junctional breakdown. Eur Spine J Off Publ Eur Spine Soc Eur Spinal Deform Soc Eur Sect Cerv Spine Res Soc 27:139-148. https://doi.org/10.1007/ s00586-017-5426-7

14. Roussouly P, Berthonnaud E, Dimnet J (2003) Geometrical and mechanical analysis of lumbar lordosis in an asymptomatic population: proposed classification. Rev Chir Orthop Reparatrice Appar Mot 89:632-639

15. Jackson RP, McManus AC (1994) Radiographic analysis of sagittal plane alignment and balance in standing volunteers and patients with low back pain matched for age, sex, and size. A prospective controlled clinical study. Spine 19:1611-1618

16. Roussouly P, Pinheiro-Franco JL (2011) Sagittal parameters of the spine: biomechanical approach. Eur Spine J Off Publ Eur Spine Soc Eur Spinal Deform Soc Eur Sect Cerv Spine Res Soc 20(Suppl 5):578-585. https://doi.org/10.1007/s00586-011-1924-1

17. Pesenti S, Lafage R, Stein D et al (2018) The Amount of Proximal Lumbar Lordosis Is Related to Pelvic Incidence. Clin Orthop 476:1603-1611. https://doi.org/10.1097/CORR.00000 00000000380

18. Berthonnaud E, Dimnet J, Roussouly P, Labelle H (2005) Analysis of the sagittal balance of the spine and pelvis using shape and orientation parameters. J Spinal Disord Tech 18:40-47

19. Schwab F, Lafage V, Patel A, Farcy J-P (2009) Sagittal plane considerations and the pelvis in the adult patient. Spine 34:1828-1833. https://doi.org/10.1097/BRS.0b013e3181a13c08

20. Barrey C, Jund J, Noseda O, Roussouly P (2007) Sagittal balance of the pelvis-spine complex and lumbar degenerative diseases. A comparative study about 85 cases. Eur Spine J Off Publ Eur Spine Soc Eur Spinal Deform Soc Eur Sect Cerv Spine Res Soc 16:1459-1467. https://doi.org/10.1007/s00586-006-0294-6

21. Battié MC, Videman T, Parent E (2004) Lumbar disc degeneration: epidemiology and genetic influences. Spine 29:2679-2690

22. Amabile C, Le Huec J-C, Skalli W (2018) Invariance of headpelvis alignment and compensatory mechanisms for asymptomatic adults older than 49 years. Eur Spine J 27:458-466. https ://doi.org/10.1007/s00586-016-4830-8 
23. Barrey C, Roussouly P, Le Huec J-C et al (2013) Compensatory mechanisms contributing to keep the sagittal balance of the spine. Eur Spine J Off Publ Eur Spine Soc Eur Spinal Deform Soc Eur Sect Cerv Spine Res Soc 22(Suppl 6):S834-S841. https ://doi.org/10.1007/s00586-013-3030-z

24. Faundez A, Cogniet A, Racloz G, Tsoupras A, Le Huec JC (2016) Spondylolisthésis dégénératif lombaire. EMC 12(1):1-7

25. Vanneuville G, Garcier JM, Poumarat G et al (1992) Mechanisms of orientation of the pelvifemoral base during static loading of the lumbar spine in weight-lifters. Surg Radiol Anat SRA $14: 29-33$

26. Legaye J, Duval-Beaupère G (2005) Sagittal plane alignment of the spine and gravity: a radiological and clinical evaluation. Acta Orthop Belg 71:213-220

27. Hovorka I, Rousseau P, Bronsard N et al (2008) Extension reserve of the hip in relation to the spine: comparative study of two radiographic methods. Rev Chir Orthop Reparatrice Appar Mot 94:771-776. https://doi.org/10.1016/j.rco.2008.03.033

28. Lazennec JY, Brusson A, Folinais D et al (2015) Measuring extension of the lumbar-pelvic-femoral complex with the $\operatorname{EOS}^{\circledR}$ system. Eur J Orthop Surg Traumatol Orthop Traumatol 25:1061-1068. https://doi.org/10.1007/s00590-015-1603-8

29. Ferrero E, Liabaud B, Challier V et al (2016) Role of pelvic translation and lower-extremity compensation to maintain gravity line position in spinal deformity. J Neurosurg Spine 24:436-446. https ://doi.org/10.3171/2015.5.SPINE14989

30. Petit M, Thompson W, Lacroix P-M, et al (2018) Équilibre sagittal du rachis: description et applications. Wwwem-Premiumcomdatatraitesap15-71722

31. Faro FD, Marks MC, Pawelek J, Newton PO (2004) Evaluation of a functional position for lateral radiograph acquisition in adolescent idiopathic scoliosis. Spine 29:2284-2289

32. Dubousset J, Charpak G, Dorion I et al (2005) A new 2D and 3D imaging approach to musculoskeletal physiology and pathology with low-dose radiation and the standing position: the EOS system. Bull Acad Natl Med 189:287-297 discussion 297-300

33. Gelb DE, Lenke LG, Bridwell KH et al (1995) An analysis of sagittal spinal alignment in 100 asymptomatic middle and older aged volunteers. Spine 20:1351-1358

34. Glassman SD, Bridwell K, Dimar JR et al (2005) The impact of positive sagittal balance in adult spinal deformity. Spine 30:2024-2029

35. Diebo BG, Varghese JJ, Lafage R et al (2015) Sagittal alignment of the spine: What do you need to know? Clin Neurol Neurosurg 139:295-301. https://doi.org/10.1016/j.clineuro.2015.10.024

36. Debarge R, Demey G, Roussouly P (2010) Radiological analysis of ankylosing spondylitis patients with severe kyphosis before and after pedicle subtraction osteotomy. Eur Spine J Off Publ Eur Spine Soc Eur Spinal Deform Soc Eur Sect Cerv Spine Res Soc 19:65-70. https://doi.org/10.1007/s00586-009-1158-7

37. Protopsaltis T, Schwab F, Bronsard N et al (2014) TheT1 pelvic angle, a novel radiographic measure of global sagittal deformity, accounts for both spinal inclination and pelvic tilt and correlates with health-related quality of life. J Bone Joint Surg Am 96:1631-1640. https://doi.org/10.2106/JBJS.M.01459

38. Boissière L, Takemoto $\mathrm{M}$, Bourghli A et al (2017) Global tilt and lumbar lordosis index: two parameters correlating with healthrelated quality of life scores-but how do they truly impact disability? Spine J Off J North Am Spine Soc 17:480-488. https:// doi.org/10.1016/j.spinee.2016.10.013

39. Le Huec JC, Leijssen P, Duarte M, Aunoble S (2011) Thoracolumbar imbalance analysis for osteotomy planification using a new method: FBI technique. Eur Spine J Off Publ Eur Spine Soc Eur Spinal Deform Soc Eur Sect Cerv Spine Res Soc 20(Suppl 5):669-680. https://doi.org/10.1007/s00586-011-1935-y
40. Amabile $\mathrm{C}$, Pillet $\mathrm{H}$, Lafage $\mathrm{V}$ et al (2016) A new quasi-invariant parameter characterizing the postural alignment of young asymptomatic adults. Eur Spine J Off Publ Eur Spine Soc Eur Spinal Deform Soc Eur Sect Cerv Spine Res Soc 25:3666-3674. https:// doi.org/10.1007/s00586-016-4552-y

41. Berjano P, Cecchinato R, Damilano M et al (2013) Preoperative calculation of the necessary correction in sagittal imbalance surgery: validation of three predictive methods. Eur Spine J Off Publ Eur Spine Soc Eur Spinal Deform Soc Eur Sect Cerv Spine Res Soc 22(Suppl 6):S847-S852. https://doi.org/10.1007/s0058 6-013-3025-9

42. Glattes RC, Bridwell KH, Lenke LG et al (2005) Proximal junctional kyphosis in adult spinal deformity following long instrumented posterior spinal fusion: incidence, outcomes, and risk factor analysis. Spine 30:1643-1649

43. Kim YJ, Bridwell KH, Lenke LG et al (2005) Proximal junctional kyphosis in adolescent idiopathic scoliosis following segmental posterior spinal instrumentation and fusion: minimum 5-year follow-up. Spine 30:2045-2050

44. Denis F, Sun EC, Winter RB (2009) Incidence and risk factors for proximal and distal junctional kyphosis following surgical treatment for Scheuermann kyphosis: minimum five-year follow-up. Spine 34:E729-E734. https://doi.org/10.1097/BRS.0b013e3181 ae2ab2

45. Bridwell KH, Lenke LG, Cho SK et al (2013) Proximal junctional kyphosis in primary adult deformity surgery: evaluation of 20 degrees as a critical angle. Neurosurgery 72:899-906. https://doi. org/10.1227/NEU.0b013e31828bacd8

46. Kim YJ, Lenke LG, Bridwell KH et al (2007) Proximal junctional kyphosis in adolescent idiopathic scoliosis after 3 different types of posterior segmental spinal instrumentation and fusions: incidence and risk factor analysis of 410 cases. Spine 32:2731-2738. https://doi.org/10.1097/BRS.0b013e31815a7ead

47. Park P, Garton HJ, Gala VC et al (2004) Adjacent segment disease after lumbar or lumbosacral fusion: review of the literature. Spine 29:1938-1944

48. Shiba Y, Taneichi H, Inami S et al (2016) Dynamic global sagittal alignment evaluated by three-dimensional gait analysis in patients with degenerative lumbar kyphoscoliosis. Eur Spine J Off Publ Eur Spine Soc Eur Spinal Deform Soc Eur Sect Cerv Spine Res Soc 25:2572-2579. https://doi.org/10.1007/s00586-016-4648-4

49. Lee CS, Lee CK, Kim YT et al (2001) Dynamic sagittal imbalance of the spine in degenerative flat back: significance of pelvic tilt in surgical treatment. Spine 26:2029-2035

50. Bae J, Theologis AA, Jang J-S et al (2017) Impact of fatigue on maintenance of upright posture: dynamic assessment of sagittal spinal deformity parameters after walking 10 minutes. Spine 42:733-739. https://doi.org/10.1097/BRS.0000000000001898

51. Diebo BG, Henry J, Lafage V, Berjano P (2015) Sagittal deformities of the spine: factors influencing the outcomes and complications Eur Spine J. 24(Suppl 1):S3-15. https://doi.org/10.1007/ s00586-014-3653-8 Epub 2014 Nov 12

52. Kobayashi T, Atsuta Y, Matsuno T, Takeda N (2004) A longitudinal study of congruent sagittal spinal alignment in an adult cohort. Spine 29:671-676

53. Kim Y-C, Lenke LG, Lee S-J et al (2017) The cranial sagittal vertical axis (CrSVA) is a better radiographic measure to predict clinical outcomes in adult spinal deformity surgery than the C7 SVA: a monocentric study. Eur Spine J Off Publ Eur Spine Soc Eur Spinal Deform Soc Eur Sect Cerv Spine Res Soc 26:21672175. https://doi.org/10.1007/s00586-016-4757-0

Publisher's Note Springer Nature remains neutral with regard to jurisdictional claims in published maps and institutional affiliations. 